\title{
Utilização de modelos unicaracterística e multicaracterística na estimação de parâmetros genéticos na raça Nelore
}

\author{
[Utilization of single-trait and multi-trait models applied to genetic parameter \\ estimation in Nellore Cattle] \\ V.B. Pedrosa ${ }^{1}$, J.P. Eler $^{2}$, J.B.S. Ferraz ${ }^{2}$, L.F.B. Pinto ${ }^{3}$ \\ ${ }^{1}$ Universidade Estadual de Ponta Grossa - UEPG - Ponta Grossa, PR \\ ${ }^{2}$ Universidade de São Paulo - USP - Pirassununga, SP \\ ${ }^{3}$ Universidade Federal da Bahia - Escola de Medicina Veterinária - Salvador, BA \\ RESUMO
}

\begin{abstract}
Estimativas dos parâmetros genéticos e dos componentes de covariância para peso ao nascimento (PN), peso à desmama (PD), circunferência escrotal (CE), ganho de peso pós-desmama (GP) e musculosidade (MUSC) foram obtidas com modelos unicaracterística, bicaracterística e multicaracterística, utilizando-se um banco de dados de 69.525 animais da raça Nelore. Os parâmetros foram estimados pelo método da máxima verossimilhança restrita, com modelos que incluíram o efeito genético aditivo direto e materno, além do grupo de manejo a desmama e de ambiente permanente da vaca, todos como aleatórios, e do efeito fixo de grupo de contemporâneo. Os coeficientes de herdabilidade estimados para as características pós-desmama com o modelo multicaracterística $(0,45 ; 0,31$ e 0,25 , respectivamente, para CE, GP e MUSC) foram maiores que as estimativas obtidas com os modelos unicaracterística $(0,42 ; 0,21$ e 0,22$)$ e bicaracterística $(0,39 ; 0,25$ e 0,24). Da mesma forma, as correlações genéticas estimadas foram, em grande parte, maiores para o modelo multicaracterística. Estes resultados indicam que a redução do viés, proporcionada pelo modelo multicaracterística, aumenta as estimativas dos parâmetros genéticos e demonstra que os resultados com análises unicaracterística e bicaracterística são subestimados. Os resultados da resposta à seleção demonstraram que a musculosidade apresentou grande potencial seletivo, e o cálculo das respostas correlacionadas de musculosidade e demais características estudadas demonstrou que sua utilização em programas de seleção pode ser vantajosa aos criadores.
\end{abstract}

Palavras-chave: bovinos, correlação genética, herdabilidade, seleção

\begin{abstract}
Genetic parameters and components of (co)variance for birth weight (BW), weaning weight (WW), scrotal circumference (SC), weight gain at post-weaning (WG), and muscle score (MUSC) were obtained with univariate, bivariate and multivariate models, using a dataset of 69,525 Nellore cattle. The parameters were estimated by restricted maximum likelihood method. Direct and maternal additive genetic effects, group of management, and maternal permanent environmental effect were adjusted as random effects, while contemporary group was adjusted as fixed effect. Multivariate approach allowed higher estimate heritability for postweaning traits $(0.45 ; 0.31$ and 0.25 , respectively for SC, WG and MUSC) than univariate $(0,42 ; 0,21$ and 0,22) and bivariate models $(0,39 ; 0,25$ and 0,24$)$. Similarly, the genetic correlations were higher in the multivariate approach. These results indicate that reducing the bias provided by the mutivariate model improves the estimates of genetic parameters and shows that the results of bivariate and univarite models are underestimated. Selection for muscle score presented great potential for correlated response in other traits and its use in selection programs can be a useful tool for breeders.
\end{abstract}

Keywords: bovine, genetic correlation, heritability, selection

Recebido em 12 de setembro de 2013

Aceito em 18 de dezembro de 2013

E-mail: vbpedrosa@uepg.br 


\section{INTRODUÇÃO}

Em bovinos de corte, as características associadas ao crescimento e produção de carne, como peso ao nascer, peso à desmama, ganho de peso pós-desmama, musculosidade e, ainda, relacionadas com a reprodução, como circunferência escrotal, são importantes para julgar a eficiência dos sistemas de produção e são constantemente consideradas como critério de seleção em programas de melhoramento genético.

Contudo, no passado, devido à grande demanda computacional ocasionada pela necessidade de resolução de complexos modelos matemáticos, análises unicaracterística e bicaracterística eram constantemente realizadas pelos geneticistas para estimar os componentes de (co)variância e parâmetros genéticos (Henderson, 1984; Meyer et al., 1993). Porém, ainda nos dias atuais, mesmo com a existência de processadores mais rápidos e capazes de realizar cálculos complexos de forma rápida, estes modelos (uni e bicaracterísticos) ainda são muito utilizados e não foram completamente substituídos por modelos multicaracterísticos, capazes de representar melhor a realidade fisiológica dos animais (Ramírez-Valverde et al., 2007).

Estimativas cada vez mais acuradas dos componentes de (co)variância têm sido exigidas, tendo em vista que as variâncias genéticas e residuais são utilizadas para estimar os coeficientes de herdabilidade, que, por sua vez, podem fornecer informações sobre a eficácia da seleção genética na alteração das características de um rebanho. Conhecer as covariâncias precisas entre as características permite selecionar mais eficientemente para o aumento ou ganho no sentido desejado da seleção (Walter e Mao, 1985). Além disso, métodos de estimação de componentes de covariância requerem que todas as informações disponíveis sejam consideradas conjuntamente para reduzir o viés nos resultados, principalmente em características expressas precocemente na vida do animal, já que fisiologicamente muitas delas são interdependentes e possuem genes em comum; portanto, não deveriam ser consideradas isoladamente.

Algumas pesquisas têm demonstrado vantagens da utilização de modelos multicaracterística de análise em comparação aos métodos unicaracterística e bicaracterística, por melhor representarem os resultados de seleção em rebanhos comerciais de gado de corte, já que esses consideram diversas características conjuntamente para a formação dos índices de seleção e fatores de descarte (Ramírez-Valverde et al., 2007; Boligon et al., 2009). Por essa razão, com o intuito de oferecer subsídios aos selecionadores, o objetivo deste estudo foi comparar os resultados das estimativas de parâmetros genéticos utilizando-se modelos multicaracterística, bicaracterística e unicaracterística para as características de interesse econômico em animais da raça Nelore, além de estimar o efeito da resposta correlacionada à seleção.

\section{MATERIAL E MÉTODOS}

Os dados utilizados na presente pesquisa são provenientes dos arquivos da Agro-Pecuária CFM Ltda., com fazendas localizadas no Sudeste e Centro-Oeste do Brasil. O banco de dados inicial continha 71.314 animais. Posteriormente, foram eliminadas as informações pertencentes a grupos de contemporâneos com menos de cinco animais, assim como grupos de contemporâneos nos quais os animais eram filhos de um único touro.

Após edição dos dados, analisaram-se os registros de 69.525 animais nascidos desde 1984 até 2009, para a estimativa dos parâmetros genéticos das características $\mathrm{PN}, \mathrm{PD}, \mathrm{CE}, \mathrm{GP}$ e MUSC. Dessas análises, foram obtidas as estatísticas descritivas, além dos coeficientes de assimetria e curtose para averiguação da normalidade das distribuições, por meio de procedimentos do programa SAS (Statistical Analysis System, 2008). As informações de pedigree foram compostas por 165.539 registros, sendo 61.249 machos e 104.290 fêmeas. A descrição do arquivo de dados utilizado é apresentada na Tab. 1.

As informações fenotípicas foram mensuradas nas diferentes fases de desenvolvimento animal, em que PN foi registrado no momento pós-parto; PD, aos 205 dias de idade; CE, aos 18 meses de idade, medida por meio de fita métrica; GP, entre a desmama e o sobreano; e MUSC, aos 18 meses de idade, atribuído escore individual, através de avaliação visual, variando de 1 a 6 , em que a maior pontuação representa o grau mais favorável. 
Tabela 1. Estatística descritiva das características, peso ao nascimento (PN), peso à desmama (PD), circunferência escrotal (CE), ganho de peso da desmama ao sobreano (GP) e musculosidade (MUSC)

\begin{tabular}{lcccccccc}
\hline \multicolumn{1}{c}{ Características } & N & Média & CV & DP & Mínimo & Máximo & Ass & Curt \\
\hline PN (kg) & 66113 & 31,43 & 12 & 3,62 & 13,00 & 58,00 & 0,22 & 0,87 \\
PD (kg) & 68553 & 190,09 & 14 & 27,04 & 61,00 & 317,00 & $-0,28$ & 1,12 \\
CE (cm) & & & & & & & & \\
& 29810 & 27,23 & 13 & 3,42 & 11,00 & 40,00 & 0,07 & 0,29 \\
GP (kg) & & & & & & & & \\
& 58660 & 114,45 & 27 & 31,39 & 10,50 & 294,54 & $-0,19$ & 0,66 \\
MUSC (escore) & 58272 & 3,68 & 25 & 0,91 & 1,00 & 6,00 & 0,17 & 0,47 \\
\hline
\end{tabular}

$\mathrm{N}$ - número de observações; CV - coeficiente de variação; DP - desvio-padrão; Ass - Assimetria; Curt - Curtose.

As análises foram realizadas no laboratório do Grupo de Melhoramento Animal e Biotecnologia da Faculdade de Zootecnia e Engenharia de Alimentos da Universidade de São Paulo (GMAB - FZEA / USP). Para estimar os parâmetros genéticos, utilizou-se a metodologia de modelos mistos sob modelo animal, por meio do software VCE 6.0 (Groeneveld et al., 2008). A análise completa do pedigree foi realizada pelo software PopReport (Groeneveld et al., 2009).

Para análise de peso ao nascimento e peso à desmama, foram incluídos como covariáveis a idade do animal na data da medição, a data Juliana de nascimento, a idade da mãe ao parto, além do efeito fixo de grupo contemporâneo. Os efeitos genéticos aditivo direto e materno, além do efeito de ambiente permanente da vaca, foram considerados aleatórios.

Para análise de circunferência escrotal, ganho de peso pós-desmama e musculosidade, foram incluídas como covariáveis a idade do animal na data da medição, a data Juliana de nascimento, a idade da mãe ao parto, além do efeito fixo de grupo contemporâneo. Os efeitos genético aditivo direto e materno foram ajustados como aleatórios, exceto para MUSC, que não considerou o efeito materno por não ter apresentado efeito significativo para essa característica em análises prévias.

Em notação matricial, o modelo geral utilizado neste estudo pode ser apresentado como segue:

$y=X \beta+Z g+M m+W w+P p+e$

Onde $\boldsymbol{y}$ é um vetor $\mathrm{N} \times 1$ de registros; $\beta$ denota o vetor de efeitos fixos; $\mathrm{X}$ é a matriz que associa $\beta$ com $\boldsymbol{y} ; \boldsymbol{g}$ é o vetor de efeitos genéticos diretos; $\mathrm{Z}$ é a matriz que associa $\boldsymbol{g}$ com Y; $\boldsymbol{m}$ é o vetor de efeito genético materno; $M$ é a matriz que associa $\boldsymbol{m}$ com $\boldsymbol{y} ; \boldsymbol{w}$ é o vetor dos efeitos de grupo de manejo à desmama; $\mathrm{W}$ é a matriz que associa $\boldsymbol{w} \operatorname{com} \boldsymbol{y} ; \boldsymbol{p}$ é o vetor do efeito de ambiente permanente mais os efeitos genéticos não aditivos atribuídos pelas mães à sua progênie; P é a matriz que associa $\boldsymbol{p} \operatorname{com} \boldsymbol{y}$; enquanto $\boldsymbol{e}$ é o vetor de efeitos residuais referentes às observações que não são explicadas por outras partes do modelo.

As variâncias e covariâncias genéticas aditivas e residuais foram estimadas pelo método da máxima verossimilhança restrita (REML) em análises unicaracterística, bicaracterística e multicaracterística. Cabe ressaltar que, em referência à MUSC, mesmo sendo esta uma característica categórica, tem sido avaliada através de modelos lineares, como reportado por Kippert et al. (2006) e Faria et al. (2008).

Para a análise multicaracterística, a representação do modelo, admitindo-se que $\mathrm{E}[\mathrm{y}]=\mathrm{X} \beta$ foi:

$$
\operatorname{Var}\left[\begin{array}{c}
\mathrm{g} \\
\mathrm{m} \\
\mathrm{w} \\
\mathrm{p} \\
\mathrm{e}
\end{array}\right]=\left[\begin{array}{ccccc}
\mathrm{A} \sigma_{a}^{2} & 0 & 0 & 0 & 0 \\
0 & \mathrm{~A} \sigma_{\mathrm{m}}^{2} & 0 & 0 & 0 \\
0 & 0 & I \sigma_{w}^{2} & 0 & 0 \\
0 & 0 & 0 & \mathrm{I} \sigma_{\mathrm{p}}^{2} & 0 \\
0 & 0 & 0 & 0 & \mathrm{I} \sigma_{\mathrm{e}}^{2}
\end{array}\right]
$$

Em que $\sigma_{\mathrm{a}}^{2}=$ variância genética aditiva direta; $\sigma_{\mathrm{m}}{ }^{2}=$ variância genética aditiva maternag ${ }_{\mathrm{w}}{ }^{2}=$ variância de grupo de manejo à desmama; $\sigma_{\mathrm{p}_{2}}{ }^{2}=$ variância de ambiente permanente; $\sigma_{\mathrm{p}}^{2}=$ variância residual. A matriz de (co)variância para os efeitos genéticos é $\mathrm{G}=\mathrm{G}_{0} \otimes \mathrm{A}$, onde A é a matriz de parentesco, $\otimes$ denota o produto direto entre as matrizes e $\mathrm{G}_{0}$ é a matriz (de ordem 9), como segue: 


$$
\mathrm{G}_{0}=\left[\begin{array}{ccccccccc}
\sigma_{g 1}^{2} & \sigma_{g 1,2} & \sigma_{g 1,3} & \sigma_{g 1,4} & \sigma_{g 1,5} & \sigma_{g m 1,1} & \sigma_{g m 1,2} & \sigma_{g m 1,3} & \sigma_{g m 1,4} \\
\sigma_{g 1,2} & \sigma_{g 2}^{2} & \sigma_{g 2,3} & \sigma_{g 2,4} & \sigma_{g 2,5} & \sigma_{g m 2,1} & \sigma_{g m 2,2} & \sigma_{g m 2,3} & \sigma_{g m 2,4} \\
\sigma_{g 1,3} & \sigma_{g 2,3} & \sigma_{g 3}^{2} & \sigma_{g 3,4} & \sigma_{g 3,5} & \sigma_{g m 3,1} & \sigma_{g m 3,2} & \sigma_{g m 3,3} & \sigma_{g m 3,4} \\
\sigma_{g 1,4} & \sigma_{g 2,4} & \sigma_{g 3,4} & \sigma_{g 4}^{2} & \sigma_{g 4,5} & \sigma_{g m 4,1} & \sigma_{g m 4,2} & \sigma_{g m 4,3} & \sigma_{g m 4,4} \\
\sigma_{g 1,5} & \sigma_{g 2,5} & \sigma_{g 3,5} & \sigma_{g 4,5} & \sigma_{g 5}^{2} & \sigma_{g m 5,1} & \sigma_{g m 5,2} & \sigma_{g m 5,3} & \sigma_{g m 5,4} \\
\sigma_{g m 1,1} & \sigma_{g m 2,1} & \sigma_{g m 3,1} & \sigma_{g m 4,1} & \sigma_{g m 5,1} & \sigma_{m 1}^{2} & \sigma_{m 1,2} & \sigma_{m 1,3} & \sigma_{m 1,4} \\
\sigma_{g m 1,2} & \sigma_{g m 2,2} & \sigma_{g m 3,2} & \sigma_{g m 4,2} & \sigma_{g m 5,2} & \sigma_{m 1,2} & \sigma_{m 2}^{2} & \sigma_{m 2,3} & \sigma_{m 2,4} \\
\sigma_{g m 1,3} & \sigma_{g m 2,3} & \sigma_{g m 3,3} & \sigma_{g m 4,3} & \sigma_{g m 5,3} & \sigma_{m 1,3} & \sigma_{m 2,3} & \sigma_{m 3}^{2} & \sigma_{m 3,4} \\
\sigma_{g m 1,4} & \sigma_{g m 2,4} & \sigma_{g m 3,4} & \sigma_{g m 4,4} & \sigma_{g m 5,4} & \sigma_{m 1,4} & \sigma_{m 2,4} & \sigma_{m 3,4} & \sigma_{m 4}^{2}
\end{array}\right]
$$

A matriz de (co)variância para o efeito de grupo de manejo à desmama é $\mathrm{W}=\mathrm{W}_{0} \otimes \mathrm{I}_{\mathrm{W}}$, onde $\mathrm{I}_{\mathrm{W}}$ é a matriz identidade de ordem apropriada para os grupos de manejo, $\otimes$ denota o produto direto entre as matrizes e $\mathrm{W}_{0}$ é a matriz (de ordem 3 ). A matriz de (co)variância para o efeito de ambiente permanente é $\mathrm{P}=\mathrm{P}_{0} \otimes_{\mathrm{C}}$, onde $\mathrm{I}_{\mathrm{C}}$ é a matriz identidade de ordem apropriada para as $\mathbf{c}$ vacas e $\mathrm{P}_{0}$ é a matriz (de ordem 2), utilizadas para peso ao nascer e peso à desmama, e $\otimes$ denota o produto direto entre as matrizes. Os subscritos 1 , 2, 3, 4 e 5 representam Peso ao Nascimento, Peso à Desmama, Circunferência Escrotal, Ganho de Peso pós-desmama e Musculosidade, respectivamente.

$\mathrm{G}_{1}=\left[\begin{array}{ccccccccc}\sigma_{g 1}^{2} & \sigma_{g 1,2} & 0 & 0 & 0 & 0 & 0 & 0 & 0 \\ \sigma_{g 1,2} & \sigma_{g 2}^{2} & \sigma_{g 2,3} & \sigma_{g 2,4} & \sigma_{g 2,5} & \sigma_{g m 2,1} & \sigma_{g m 2,2} & \sigma_{g m 2,3} & \sigma_{g m 2,5} \\ 0 & \sigma_{g 2,3} & \sigma_{g 3}^{2} & 0 & 0 & 0 & 0 & 0 & 0 \\ 0 & \sigma_{g 2,4} & 0 & \sigma_{g 4}^{2} & 0 & 0 & 0 & 0 & 0 \\ 0 & \sigma_{g 2,5} & 0 & 0 & \sigma_{g 5}^{2} & 0 & 0 & 0 & 0 \\ 0 & \sigma_{g m 2,1} & 0 & 0 & 0 & \sigma_{m 1}^{2} & \sigma_{m 1,2} & 0 & 0 \\ 0 & \sigma_{g m 2,2} & 0 & 0 & 0 & \sigma_{m 1,2} & \sigma_{m 2}^{2} & \sigma_{m 2,3} & \sigma_{m 2,5} \\ 0 & \sigma_{g m 2,3} & 0 & 0 & 0 & 0 & \sigma_{m 2,3} & \sigma_{m 3}^{2} & 0 \\ 0 & \sigma_{g m 2,5} & 0 & 0 & 0 & 0 & \sigma_{m 2,5} & 0 & \sigma_{m 5}^{2}\end{array}\right]$

Para o presente estudo, representação semelhante pode ser descrita para a matriz de (co)variância dos efeitos de grupo de manejo à desmama como
Para o modelo bicaracterística, peso à desmama foi considerado uma característica âncora nas análises, devido ao fato de conter maior número de informações que determinam as decisões de seleção, tal como mencionado por Meyer et al. (1993). No entanto, se os critérios de seleção consistem em cinco características em conjunto, a matriz de (co)variância para efeitos genéticos é $\mathrm{G}=\mathrm{G}_{1} \otimes \mathrm{A}$, onde A é a matriz de parentesco, $\otimes$ denota o produto cruzado e $G_{1}$ é a matriz (de ordem 9) configurada de zeros nos valores de covariância que não são relacionadas com PD da seguinte forma:

$$
\text { respectivamente. }
$$

$\mathrm{W}=\mathrm{W}_{1} \otimes \mathrm{I}_{\mathrm{W}}$, onde $\mathrm{I}_{\mathrm{W}}$ é a matriz identidade de ordem apropriada para $\mathbf{w}$ grupos de manejo, $\otimes$ 
denota o produto direto entre as matrizes e $\mathrm{W}_{1}$ é a matriz (de ordem 3) configurada de valores zero para todos as covariâncias consideradas, uma vez que, para o modelo bicaracterística com peso à desmama como característica âncora, os efeitos mencionados não são correlacionados. A matriz de (co)variância para os efeitos de ambiente permanente (P1) do modelo bicaracterística é igual àquela considerada na análise multicaracterística, com exceção das covariâncias que não estão correlacionados ao peso à desmama, que configuram valor igual a zero.

O progresso genético, ou resposta à seleção, foi calculado para as características em estudo por $\Delta \mathrm{G}=\mathrm{i} \sigma_{\mathrm{p}} \mathrm{h}^{2}$, em que: $\Delta \mathrm{G}=$ progresso genético ou resposta à seleção por geração de seleção; i = intensidade de seleção na característica; $\sigma_{\mathrm{p}}=$ desvio-padrão fenotípico da característica; $h^{2}=$ herdabilidade da característica.

Posteriormente foi realizado o cálculo da resposta correlacionada utilizando-se $\mathrm{RC}_{\mathrm{Y}}=\mathrm{i}_{\mathrm{X}} \mathrm{h}_{\mathrm{X}} \mathrm{rg}_{\mathrm{xy}} \sigma_{\mathrm{gy}}$, em que: $\mathrm{RC}_{\mathrm{Y}}=$ resposta correlacionada em $\mathrm{Y} ; \mathrm{i}_{\mathrm{x}}=$ intensidade de seleção aplicada em $\mathrm{X} ; \mathrm{h}_{\mathrm{x}}=$ raiz quadrada da herdabilidade de $\mathrm{X} ; \operatorname{rg}_{\mathrm{xy}}=$ correlação genética entre $\mathrm{X}$ e $\mathrm{Y} ; \sigma_{\mathrm{gy}}=$ desvio-padrão genético de $\mathrm{Y}$. No presente estudo, para realização da simulação de resposta correlacionada, foram utilizadas como critério de seleção as características que apresentaram maior capacidade de resposta à seleção.

\section{RESULTADOS E DISCUSSÃO}

As estimativas de variâncias genéticas aditivas, residuais e fenotípicas são apresentadas na Tab. 2, respectivamente, para as características PN, PD, CE, GP e MUSC. As variâncias genéticas aditivas das análises multicaracterística foram superiores às estimadas nas análises unicaracterística e bicaracterística, principalmente para as características medidas ao sobreano. Esse aumento na diferença do componente de variância genético aditivo reflete a redução do viés existente nas análises que consideram apenas o desempenho de uma característica individualmente ou que consideram somente duas características como critério de avaliação (Meyer, 1991).

Tabela 2. Estimativas de variância e co-variância para as características, peso ao nascimento (PN), peso à desmama (PD), circunferência escrotal (CE), ganho de peso da desmama ao sobreano (GP) e musculosidade (MUSC)

\begin{tabular}{|c|c|c|c|c|c|c|}
\hline & & $\mathrm{PN}$ & PD & $\mathrm{CE}$ & GP & MUSC \\
\hline \multirow{6}{*}{ UNI } & $\sigma_{a}^{2}$ & 3,20 & 84,20 & 2,70 & 80,20 & 0,11 \\
\hline & $\sigma_{m}^{2}$ & 0,65 & 25,80 & 0,10 & 29,10 & - \\
\hline & $\sigma_{\text {pe }}^{2}$ & 0,03 & 23,90 & - & - & - \\
\hline & $\sigma^{2}{ }_{e}^{2 x}$ & 5,80 & 189,50 & 3,50 & 263,20 & 0,4 \\
\hline & $\sigma_{\mathrm{a}, \mathrm{m}}$ & 0,01 & 15,90 & 0,05 & 8,65 & - \\
\hline & $\sigma_{p}^{2}$ & 9,70 & 355,20 & 6,40 & 389,80 & 0,53 \\
\hline \multirow{6}{*}{ BI } & $\sigma_{a}^{2}$ & 3,20 & 84,20 & 2,70 & 101,70 & 0,13 \\
\hline & $\sigma_{\mathrm{m}}^{2}$ & 0,70 & 24,90 & 0,85 & 32,90 & - \\
\hline & $\sigma_{\text {pe }}^{2}$ & 0,16 & 24,20 & - & - & - \\
\hline & $\sigma_{e}^{2}$ & 5,8 & 189,50 & 3,70 & 261,40 & 0,4 \\
\hline & $\sigma_{\mathrm{a}, \mathrm{m}}$ & 0,02 & 16,20 & $-0,12$ & 6,10 & - \\
\hline & $\sigma_{p}^{2,11}$ & 9,90 & 355,20 & 7,00 & 408,30 & 0,55 \\
\hline \multirow{6}{*}{ MULTI } & $\sigma_{a}^{2}$ & 3,30 & 88,20 & 3,00 & 127,20 & 0,14 \\
\hline & $\sigma_{m}^{2}$ & 0,65 & 64,40 & 0,60 & 43,70 & - \\
\hline & $\sigma_{\text {pe }}^{2}$ & 0,27 & 22,70 & - & - & - \\
\hline & $\sigma_{\mathrm{e}}^{2}$ & 6,00 & 201,40 & 3,40 & 253,60 & 0,4 \\
\hline & $\sigma_{\mathrm{a}, \mathrm{m}}$ & 0,09 & 11,25 & $-0,15$ & $-11,75$ & - \\
\hline & $\sigma_{p}^{2}$ & 10,40 & 399,20 & 6,7 & 401,00 & 0,57 \\
\hline
\end{tabular}

UNI - unicaracterística; BI - bicaracterística; MULTI - multicaracterística; $\sigma_{a}^{2}$ - variância genética aditiva direta; $\sigma_{m}^{2}$ - variância genética aditiva materna; $\sigma^{2}{ }_{\mathrm{pe}}-$ variância do efeito de ambiente permanente; $\sigma^{2}{ }_{\mathrm{e}}-$ variância residual; $\sigma_{\mathrm{a}, \mathrm{m}}$ - co-variância genética entre os efeitos direto e materno; $\sigma_{p}^{2}-$ variância fenotípica. 
Os resultados deste trabalho confirmam os achados de Mercadante et al. (2004), que observaram aumento nas estimativas de variâncias genéticas aditivas de diferentes pesos de bovinos quando avaliados em análises multicaracterística. Em outra pesquisa, avaliando pesos ao nascimento, à desmama, ao ano e à maturidade, Meyer et al. (1993) observaram aumento nas estimativas da variância genética aditiva em análises envolvendo três ou quatro pesos simultaneamente em relação às análises unicaracterística. Do mesmo modo, em trabalho mais recente, Boligon et al. (2009) analisaram animais da raça Nelore e obtiveram estimativas de variância genética aditiva superiores em análises multicaracterística se comparadas com as unicaracterística e bicaracterística, para os pesos medidos a partir do sobreano.

As estimativas da variância residual para as características pós-desmama foram mais altas nas análises unicaracterística e bicaracterística em comparação às estimadas nos modelos multicaracterística, com exceção de MUSC, que foi semelhante para os três modelos de análise. Para as características de peso ao nascimento e peso à desmama, as estimativas de variância residual obtidas nas análises unicaracterística e bicaracterística foram inferiores às da análise multicaracterística. Isso se deve à influência da pré-seleção existente no período da desmama, em que muitos animais são descartados antes de atingirem a idade ao sobreano e, consequentemente, uma possível subestimação da variância residual é aferida nos modelos uni e bicaracterística. Esse resultado indica a importância da inclusão de todas as características analisadas conjuntamente, no modelo de avaliação genética, para a definição dos critérios de seleção e descarte.

Para PN, PD e MUSC, as variâncias fenotípicas, obtidas pelo sistema de equação de modelos mistos, dos modelos unicaracterística e bicaracterística foram menores em comparação ao multicaracterística, e seguiram, portanto, as tendências das variâncias aditivas e residuais que compuseram as variâncias fenotípicas em cada modelo de análise. No entanto, para CE e GP, as variâncias fenotípicas foram menores para multicaracterística em comparação com bicaracterística, mesmo apresentando variâncias aditivas maiores em relação às análises unicaracterística e bicaracterísica. Essa diferença pode estar relacionada ao maior viés proporcionado pela utilização da metodologia bicaracterística em relação à multicaracterística (Pollak et al., 1984) e resultará em alteração nas estimativas de herdabilidade e correlação entre as características.

Colaborando com essa teoria, Meyer (1991) menciona que a análise unicaracterística resulta em um maior viés em comparação à análise multicaracterística e que esta última pode contribuir para uma seleção mais aprimorada. Deve-se destacar ainda que o viés causado pela pré-seleção de animais em idades precoces, comum em rebanhos de bovinos de corte, pode ser reduzido pela inclusão conjunta das informações das características sobre as quais a decisão de seleção foi realizada.

As estimativas de herdabilidade das análises unicaracterística resultaram em valores de 0,32, 0,23, 0,42, 0,21 e 0,22, respectivamente, para PN, PD, CE, GP e MUSC. As correlações genéticas e estimativas de herdabilidade para os modelos bicaracterística e multicaracterística estão apresentadas na Tab. 3.

Os coeficientes de herdabilidade para PN e PD nas análises unicaracterística, bicaracterística e multicaracterística foram semelhantes pelo fato de não haver seleção no período pré-desmama, o que sugere que, até a época da desmama, as estimativas de herdabilidade podem ser obtidas em análise unicaracterística. Estes resultados são condizentes com o trabalho de Meyer et al. (1993), os quais, trabalhando com modelos unicaracterística e multicaracterística, estimaram herdabilidade variando de 0,43 a 0,45 e de 0,19 a 0,21 para PN e PD, respectivamente, apresentando as maiores magnitudes para o segundo modelo mencionado. Boligon et al. (2009), ainda, obtiveram valores de 0,25 para PN e variando de 0,32 a 0,33 para PD, ambos utilizando-se análises unicaracterística, bicaracterística e multicaracterística. Esses autores mencionam que, devido a uma préseleção ao desmame, as informações das fases iniciais da vida do animal devem ser incluídas nas análises pós-desmama, para considerar o efeito dessa pré-seleção em análises conjuntas. 
Tabela 3. Coeficientes de herdabilidade dos efeitos genéticos direto e materno (diagonal) e correlação genética entre peso ao nascimento (PN), peso à desmama (PD), circunferência escrotal (CE), ganho de peso pós-desmama (GP) e musculosidade (MUSC) (acima da diagonal)

\begin{tabular}{|c|c|c|c|c|c|c|c|c|c|}
\hline \multicolumn{10}{|c|}{ Bicaracterística } \\
\hline & PN & PD & $\mathrm{CE}$ & GP & MUSC & PN_M & PD_M & CE_M & GP_M \\
\hline PN & 0,32 & 0,67 & -0.04 & 0,14 & 0,10 & $-0,11$ & $-0,13$ & $-0,10$ & $-0,08$ \\
\hline PD & - & 0,24 & 0,23 & 0,42 & 0,53 & 0,09 & 0,02 & $-0,16$ & $-0,29$ \\
\hline $\mathrm{CE}$ & - & - & 0,39 & 0,38 & 0,34 & 0,00 & 0,04 & $-0,25$ & $-0,26$ \\
\hline GP & - & - & - & 0,25 & 0,67 & 0,36 & 0,39 & 0,01 & $-0,56$ \\
\hline MUSC & - & - & - & - & 0,24 & 0,25 & 0,51 & $-0,03$ & $-0,19$ \\
\hline PN_M & - & - & - & - & - & $\mathbf{0 , 0 7}$ & 0,25 & 0,15 & $-0,22$ \\
\hline PD_M & - & - & - & - & - & - & $\mathbf{0 , 0 7}$ & 0,58 & $-0,77$ \\
\hline CE_M & - & - & - & - & - & - & - & 0,12 & $-0,11$ \\
\hline GP_M & - & - & - & - & - & - & - & - & 0,08 \\
\hline \multicolumn{10}{|c|}{ Multicaracterística } \\
\hline & PN & PD & $\mathrm{CE}$ & GP & MUSC & PN_M & PD_M & CE_M & GP_M \\
\hline $\mathrm{PN}$ & 0,31 & 0,64 & 0,01 & 0,20 & 0,18 & 0,04 & 0,05 & 0,01 & $-0,19$ \\
\hline PD & - & 0,22 & 0,25 & 0,46 & 0,56 & 0,28 & 0,22 & $-0,08$ & $-0,32$ \\
\hline CE & - & - & 0,45 & 0,35 & 0,34 & 0,08 & 0,16 & $-0,25$ & $-0,23$ \\
\hline GP & - & - & - & 0,31 & 0,76 & 0,33 & 0,55 & 0,14 & $-0,62$ \\
\hline MUSC & - & - & - & - & 0,25 & 0,29 & 0,50 & 0,05 & $-0,51$ \\
\hline PN_M & - & - & - & - & - & 0,06 & 0,24 & 0,04 & $-0,13$ \\
\hline PD_M & - & - & - & - & - & - & 0,16 & 0,31 & $-0,96$ \\
\hline CE_M & - & - & - & - & - & - & - & 0,09 & $-0,28$ \\
\hline GP_M & - & - & - & - & - & - & - & - & 0,11 \\
\hline
\end{tabular}

$\mathrm{M}$ - efeito materno.

As herdabilidades estimadas para PD estão de acordo com os valores descritos na literatura para animais da raça Nelore (Horimoto et al., 2007; Boligon et al., 2008), em que, nesses trabalhos, as estimativas variaram de 0,28 a 0,33 utilizando-se modelos bicaracterística e multicaracterística, respectivamente. As estimativas de herdabilidade materna para PN e $\mathrm{PD}$ foram semelhantes aos descritos por Eler et al. (2000), com valores entre 0,07 a 0,10, e Boligon et al. (2008) e Van Melis et al. (2010), que obtiveram estimativas entre 0,10 a 0,17 , respectivamente, para $\mathrm{PN}$ e PD. A herdabilidade materna para PD nos modelos unicaracterística e bicaracterística foi bem inferior à do modelo multicaracterística, demonstrando que parte do efeito genético materno é desconsiderado quando não se utilizam todas as características conjuntamente.

Os coeficientes de herdabilidade para as características pós-desmama foram maiores para as análises multicaracterística do que para uni e bicaracterística, ou seja, parte das estimativas de herdabilidade dessas características é subestimada quando realizada pelas metodologias unicaracterística e bicaracterística, o que, consequentemente, influencia nos cálculos de valores genéticos dos animais. Boligon et al. (2009) também observaram aumento significativo nos coeficientes de herdabilidade de características 
pós-desmama quando estas foram analisadas por metodologia multicaracterística em relação à unicaracterística e à bicaracterística. Esses autores mencionam a importância de se estimar as variâncias aditivas e residuais para cada idade considerando as correlações existentes em todas as fases da vida em estudo, ou seja, do nascimento ao sobreano. Os coeficientes de herdabilidade para CE, GP e MUSC neste estudo são similares aos valores encontrados na literatura para a raça Nelore. Para CE, Silva et al. (2006) estimaram valores de 0,46 e Van Melis et al. (2010) obtiveram herdabilidade de 0,42 para a característica mencionada, ambos trabalhando com modelos bicaracterística. Para GP, Van Melis et al. (2003 e 2010) obtiveram valores de 0,27 e 0,25 , respectivamente, em bovinos Nelore criados no Brasil. Estimativas de herdabilidade para MUSC foram reportadas por Van Melis et al. (2003 e 2010), que apresentaram 0,22 e 0,23 , respectivamente, considerando diferentes metodologias de análise.

As correlações genéticas entre as características, para os modelos bicaracterística e multicaracterística (Tab. 3) com PN foram positivas, variando entre magnitudes baixas, como entre PN e MUSC (0,10); moderadas, como entre PN e GP $(0,20)$; e altas, como entre PN e PD (0,64 e 0,67). No entanto, a correlação de PN com CE apresentou-se praticamente nula. É importante ressaltar que as correlações de PN com as características pós-desmama, utilizando o modelo multicaracterística, foram maiores em relação ao bicaracterística, demonstrando haver subestimação dos valores de correlação quando são estimadas por metodologia bicaracterística. Os resultados obtidos estão de acordo com Boligon et al. (2009), em estudo correlacionando o peso ao nascimento com outras características produtivas. Ainda, as correlações genéticas estimadas entre PD e as características pósdesmama foram altas e positivas, porém maiores quando avaliadas pelo modelo multicaracterística em comparação ao bicaracterística. Estes resultados estão de acordo com os descritos por Eler et al. (1996) e sugerem que os genes responsáveis por pesos à desmama mais elevados, em grande parte, são os mesmos que influenciam o aumento das outras características pós-desmama em questão. Cabe ressaltar que estudos do genoma bovino e das relações genotípicas entre as características devem ser realizados para comprovar tal hipótese.

As estimativas de correlações genéticas entre características pós-desmama para os modelos bicaracterística e multicaracterística foram altas e positivas, estando de acordo com os valores obtidos por Pereira et al. (2010) e Pedrosa et al. (2010). As correlações de CE com GP e MUSC foram similares para ambos os modelos analisados; no entanto, se comparadas às correlações de GP e MUSC, o valor apresentado no multicaracterística é superior em 0,09, mostrando novamente que as análises com o modelo bicaracterística resultam em valores inferiores.

O trabalho mostra que importantes diferenças nas estimativas dos parâmetros genéticos foram obtidas quando comparando o modelo multicaracterística aos demais modelos utilizados. Considerando a metodologia multicaracterística como a mais adequada, conforme demonstrado por Pollak et al. (1984), é recomendada sua utilização como ferramenta de análise para uma melhor estimação dos componentes de variância e, consequentemente, dos coeficientes de herdabilidade e correlações genéticas.

Como o modelo multicaracterística é o mais indicado para definição dos critérios de seleção, foi realizado, a partir de seus resultados, o cálculo dos progressos genéticos esperados, bem como o potencial de resposta, como percentual em relação à média da característica. Para isso, o valor 1 foi considerado como a intensidade de seleção para todas as características, o que corresponde a $40 \%$ de machos e fêmeas, e utilizou-se os desvios-padrão fenotípicos e as estimativas de herdabilidade previamente calculadas. As estimativas de progresso genético são apresentadas na Tab. 4.

Pode-se observar que a característica de maior potencial seletivo foi GP, cujo progresso genético esperado foi de 5,42\% em relação à média da característica. Muitos selecionadores escolhem seus reprodutores através de provas para o ganho de peso, sendo esta, portanto, uma característica de grande interesse econômico e que pode contribuir para melhoria de outras importantes, como PN, PD e MUSC. 
Tabela 4. Estimativas de progresso genético esperado, em valor absoluto e como percentual em relação à média, para o peso ao nascimento (PN), peso à desmama (PD), circunferência escrotal (CE), ganho de peso da desmama ao sobreano (GP) e musculosidade (MUSC)

\begin{tabular}{ccc} 
Características & $\begin{array}{c}\text { Progresso genético } \\
\text { esperado por geração }\end{array}$ & $\begin{array}{c}\text { Progresso genético esperado por geração em } \\
\text { relação à média da característica (\%) }\end{array}$ \\
\hline PN (kg) & 1,00 & 3,18 \\
PD (kg) & 4,39 & 2,30 \\
CE (cm) & 1,16 & 4,26 \\
GP (kg) & 6,20 & 5,42 \\
MUSC (escore) & 0,19 & 5,16
\end{tabular}

Outra característica de considerável potencial seletivo é MUSC, apesar de não ter sido a característica com maior estimativa de herdabilidade, apresentando um progresso genético de 5,16\% em relação à sua média por geração de seleção. A seleção para MUSC é importante por estar diretamente relacionada à produção de carne e pode trazer benefícios para PN, PD e GP. No entanto, vale ressaltar que, por ser uma característica de avaliação visual, o sucesso da seleção através desta está relacionado ao bom desempenho do avaliador que atribui o escore de musculosidade.

Da mesma maneira, CE apresentou considerável potencial seletivo e seu progresso genético esperado foi de 4,26\%. Além disso, é uma característica altamente correlacionada com importantes características de interesse econômico e mais difíceis de serem mensuradas, como, por exemplo, precocidade sexual e o ganho de peso médio diário em diferentes idades. $\mathrm{Na}$ bovinocultura de corte, a circunferência escrotal representa uma das características reprodutivas mais importantes e mais fáceis de serem mensuradas, estando relacionada com diferentes pesos e características de avaliação visual (Eler et al., 1996).

As características PN e PD apresentaram potenciais seletivos mais baixos, de 3,18 e 2,30, respectivamente. Esses resultados mostram que, apesar de muitos criadores optarem por selecionar animais para o peso à desmama, para a população em estudo, o progresso genético esperado por geração para essa característica, em relação à sua média, é mais lento, e irá influenciar o desempenho de outras características, como PN, CE, GP e MUSC.
Para melhor identificação do quanto a seleção para uma característica é capaz de alterar outra geneticamente relacionada, foi realizado 0 cálculo da resposta correlacionada, utilizando como critério de seleção as características GP e MUSC, as quais apresentaram maior capacidade de progresso genético. Foram calculadas suas respostas correlacionadas sobre $\mathrm{PN}, \mathrm{PD}$ e $\mathrm{CE}$, além de uma sobre a outra, e a intensidade de seleção considerada foi 1 , que corresponde à seleção de cerca de $40 \%$ dos machos e fêmeas do rebanho.

O estudo da resposta correlacionada de GP sobre PN, PD, CE e MUSC apresentou resultado de 0,20\%, 3,41\%, 0,32\% e 0,19\%, respectivamente, por geração. Desse modo, evidenciou-se que a seleção para GP resulta em aumento, de acordo com as magnitudes mencionadas, para as características em questão, com maior destaque para PD. Esse aspecto é importante, já que muitos animais são vendidos quando desmamados e precificados de acordo com o peso, e, portanto, uma resposta correlacionada favorável sobre essa característica é vantajosa ao criador.

Já no estudo de MUSC sobre as demais características, a maior resposta correlacionada foi com GP, em que foi identificado um aumento de $5,97 \%$ por geração quando a seleção é baseada na musculosidade. Esse resultado é importante, pois grande parte dos criadores que selecionam os animais para o aumento da qualidade de carcaça objetiva também o incremento de peso, almejando maior retorno financeiro na época de abate. A resposta de MUSC sobre PN, PD e CE foi de, respectivamente, $0,17 \%, 3,78 \%$ e $0,29 \%$. 


\section{CONCLUSÕES}

O modelo de análise multicaracterística que inclui as informações pré-desmama é o mais recomendado para avaliações genéticas de características pós-desmama por favorecer a redução do viés ocasionado pelo descarte de animais nas fases iniciais de criação. Os resultados do cálculo do progresso genético demonstram que GP apresentou o maior potencial seletivo em relação às demais características. A resposta correlacionada de GP sobre PN, PD, CE e MUSC demonstrou que a inclusão dessa característica em programas de seleção é vantajosa e pode trazer benefícios a outras características de desenvolvimento ponderal e reprodutivo.

\section{AGRADECIMENTOS}

Os autores agradecem à FAPESP pelo suporte financeiro e à Agro-Pecuária CFM Ltda. pela concessão dos dados utilizados na pesquisa.

\section{REFERÊNCIAS}

BOLIGON, A.A.; ALBUQUERQUE, L.G.; MERCADANTE, M.E.Z. et al. Herdabilidades e correlações entre pesos do nascimento à idade adulta em rebanhos da raça Nelore. Rev. Bras. Zootec., v.38, p.2320-2326, 2009.

BOLIGON, A.A.; ALBUQUERQUE, L.G.; RORATO, P.R.N. Associações genéticas entre pesos e características reprodutivas em rebanhos da raça Nelore. Rev. Bras. Zootec., v.37, p.596601, 2008.

ELER, J.P.; FERRAZ, J.B.S.; SILVA, P.R. Parâmetros genéticos para peso, avaliação visual e circunferência escrotal na raça Nelore, estimados por modelo animal. Arq. Bras. Med. Vet. Zootec., v.48, p.203-213, 1996.

ELER, J.P.; FERRAZ, J.B.S.; GOLDEN, B.L. et al. Influência da interação touro $\mathrm{x}$ rebanho na estimação da correlação entre efeitos genéticos direto e materno em bovinos da raça Nelore. Rev. Bras. Zootec., v.29, p.1642-1648, 2000.

FARIA, C.U.; MAGNABOSCO, C.U.; ALBUQUERQUE, L.G. de et al. Análise genética de escores de avaliação visual de bovinos com modelos bayesianos de limiar e linear. Pesq. Agropec. Bras., v.43, p.835-841, 2008.
GROENEVELD, E.; KOVAC, M.; MIELENZ, $\mathrm{N}$. VCE User's guide and reference manual. Version 6.0. Department of Animal Science, University of Illinois, Urbana, IL, 2008. 132p.

GROENEVELD, E.; WESTHUIZEN, B.V.D.; MAIWASHE, A. et al. POPREP: a generic report for population management. Gen. Mol. Res., v.8, p.1158-1178, 2009.

HENDERSON, C.R. Estimation of variances and covariances under multiple trait models. J. Dairy Sci., v.67, p.1581-1589, 1984.

HORIMOTO, A.R.V.R.; FERRAZ, J.B.S.; BALIEIRO, J.C.C. et al. Phenotypic and genetic correlations for body structure scores (frame) with productive traits and index for CEIP classification in Nellore beef cattle. Gen. Mol. Res., v.6, p.188-196, 2007.

KIPPERT, C.J.; RORATO, P.R.N.; CAMPOS, L.T. et al. Efeito de fatores ambientais sobre escores de avaliação visual à desmama e estimativa de parâmetros genéticos, para bezerros da raça Charolês. Cienc. Rural, v.36, p.579-585, 2006.

MERCADANTE, M.E.Z.; RAZOOK, A.G.; TROVO, J.B.F. et al. Parâmetros genéticos do peso no início da estação de monta, considerando indicativo do peso adulto de matrizes Nelore. Rev. Bras. Zootec., v.33, p.1135-1144, 2004.

MEYER, K. Estimating variances and covariances for multivariate animal models by restricted maximum likelihood. Genet. Select. Evol., v.23, p.67-83, 1991.

MEYER, K.; CARRICK, M.J.; DONNELLY, B.J.P. Genetic parameter for growth traits of Australian beef cattle from a multibreed selection experiment. J. Anim. Sci, v.71, p.2614-2622, 1993.

PEDROSA, V.B.; ELER, J.P.; SILVA, J. A. II de V. et al. Estimação dos parâmetros genéticos do peso adulto na raça Nelore. Rev. Bras. Saúde Prod. Anim., v.11, p.104-113, 2010.

PEREIRA, M.C.; YOKOO, M.J.; BIGNARDI, A.B. et al. Hip height and its relationships with reproductive and growth traits in Nelore cattle. Pesq. Agropec. Bras., v.45, p.613-620, 2010.

POLLAK, E.J.; VAN DER WERF, J.; QUAAS, R.L. Selection bias and multiple trait evaluation. J. Dairy Sci., v.67, p.1590-1595, 1984. 
RAMÍREZ-VALVERDE R.; HERNANDEZALVAREZ, O.C.; NUNEZ-DOMINGUEZ, R. et al. Análisis univariado vs multivariado en la evaluación genética de variables de crecimiento en dos razas bovinas. Agrociencia, v.41, p.271282, 2007.

STATISTICAL Analysis System - SAS. SAS user's guide. Version 9.2. Cary: 2008.

SILVA, J.A. II V.; FORMIGONI, I.B.; ELER, J.P. et al. Genetic relationship among stayability, scrotal circumference and post-weaning weight in Nelore cattle. Livest. Sci., v.99, p.51-59, 2006.
VAN MELIS, M.H.; ELER, J.P.; SILVA, J.A. II $\mathrm{V}$. et al. Estimate of genetic parameters in beef cattle using restricted maximun likelihood and method R. Rev. Bras. Zootec., v.32, p.16241632, 2003.

VAN MELIS, M.H.; OLIVEIRA, H.N.; ELER, J.P. et al. Additive genetic relationship of longevity with fertility and production traits in Nellore cattle based on bivariate models. Gen. Mol. Res., v.9, p.176-187, 2010.

WALTER, J. P.; MAO, I. L. Multiple and single trait analyses for estimating genetic parameters in simulated populations under selection. $J$. Dairy Sci. v.68, p.91-98, 1985. 